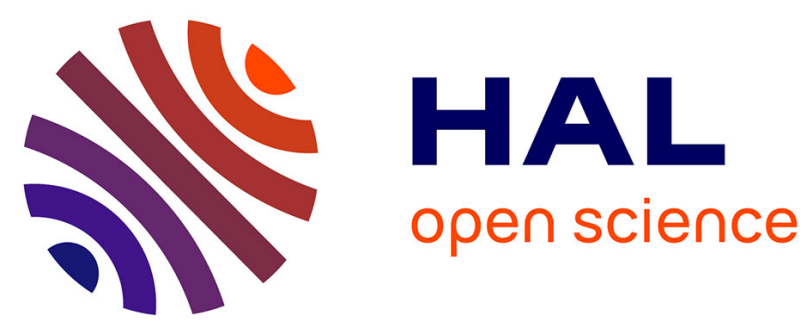

\title{
Mosaïques au Bellérophon
}

\author{
Jocelyn M.C. Toynbee
}

\section{To cite this version:}

Jocelyn M.C. Toynbee. Mosaïques au Bellérophon. Gallia - Fouilles et monuments archéologiques en France métropolitaine, 1955, 13 (1), pp.91-97. 10.3406/galia.1955.1429 . hal-01923446

\section{HAL Id: hal-01923446 \\ https://hal.science/hal-01923446}

Submitted on 6 Feb 2020

HAL is a multi-disciplinary open access archive for the deposit and dissemination of scientific research documents, whether they are published or not. The documents may come from teaching and research institutions in France or abroad, or from public or private research centers.
L'archive ouverte pluridisciplinaire HAL, est destinée au dépôt et à la diffusion de documents scientifiques de niveau recherche, publiés ou non, émanant des établissements d'enseignement et de recherche français ou étrangers, des laboratoires publics ou privés.

\section{(1)}

Distributed under a Creative Commons Attribution - NonCommercial - NoDerivatives 44.0 
(diamètre et largeur : $\bar{j}^{\mathrm{cm}}$ ) est formé de 12 méandres ( 6 de chaque côté), dont les boucles ont un écartement intérieur de $2^{\mathrm{cm}, \bar{j}}$ environ. La tige est un fil d'étain de section ovale (diamètres: $0^{\mathrm{m}}, 002$ et $\left.0^{\mathrm{m}}, 01 \overline{5}\right)$. Déchelette 3 parle de bracelets semblables. C'est probablement un modèle importé d'Italie par la voie des Alpes et dont la vogue a duré longtemps puisqu'on en a retrouvé un exemplaire parmi les trouvailles gallo-romaines de Bouta. ${ }^{4}$.

Les deux bagues ont trois spirales (diamètre : $2^{\mathrm{cm}}$ ). La section du fil est semicirculaire (largeur : $0^{\mathrm{m}}, 003$; épaisseur : $0^{\mathrm{m}}, 001$ ).

D'après l'abbé Favret.5, cinq sépultures de la nécropole de Saint-Jean sont. du premier âge du fer. La tombe de Saint-Laurent-de-la-Còte semble plus récente. La ressemblance des fibules en arbalète et à méandres avec celles décrites par Déchelette, et la complexité de l'ornementation de deux bracelets en bronze, font plutôt penser au second àge du fer, plus précisément à La Tène II.

Abbé M. Hidor:

Mosaigles at BedtérophuN

La mosaïque de Bellérophon et des Saisons découverte à Nîmes le 29 juin 1950 a fait l'objet d'une publication définitive dans Fallia par les soins de M. Jacques Aymard 1. Parmi les milliers de pave-

(4) Ch. Marteaux et Marc Le houx, Boutae (Les Fins d'Annecy), vicus gallo-romain de la cite de Vienne, Annecy, 1913, p. 104 et pl. xxiII, 9.

(5) Revue des Musées et Collections archíologriques, $\mathrm{n}^{\circ}$ 10, 1929, p. 34-44: L'age des sipultures de Saint-Jean-de-Belleville; reproduit les huit planches de la plaquette de Costa de Beauregard. ments à sujets mythologiques qui nous sont parvenus de l'Empire romain, ceux qui illustrent l'histoire de Bellérophon sont particulièrement rares. Outre celui de Nîmes, quatre autres seulement sont signalés par M. Aymard: Olynthe, $\Lambda u-$ tun, Avenches et Ravenne (Palais de Théodoric). Je voudrais ici attirer l'attention sur deux autres documents moins connus, qui complètent cette série.

L'un d'eux, découvert à Coïmbre au Portugal (Conimbriga), ne m'est connu que de seconde main, par un dessin (fig. 1)². La scène, exécutée comme à Nîmes en couleurs dans un champ circulaire (ici beaucoup plus vaste), est très fragmentaire. De Pégase il ne reste que l'avant-train, moins la tête; Bellórophon a entièrement disparu. Mais la Chimère. qui bondit vers la droite sous les jambes du cheval, est intacte : la tête, la crinière et les pattes du lion sont reprósentées avec précision, toutefois la tête qui lui sort du milieu du dos n'est pas celle d'une chèvre, mais d'un animal au nez pointu ressemblant à un loup, avec une langue effilée, des oreilles pointues, et une "fraise » touffue de fourrure autour du cou; et la queue ne se termine pas, comme elle le devrait, en tête de serpent. Ou bien le mosaïste était ignorant des éléments dont ce monstre était composé, ou bien le dessin est infidèle. Une forte ligne sous l'arrière-train de la Chimère représente son ombre; et une touffe de feuilles pointues indique le paysage. Il est remarquable que la courbe du cadre circulaire soit très large : le motif de Bellérophon ne devait occuper qu'une petite partie de ce cercle, près du bord.

I.e second pavement m'est connu di-

(1) Gallia, XI-1953, p. 249-271, fig. 1-6.

(2) Boletim da Direção Geral dos Edifícios e Monumentos Nacionais, LII-LIII : Oppidum Romano de Conimbrifa, juin-septembre 1948, fig. 52. 


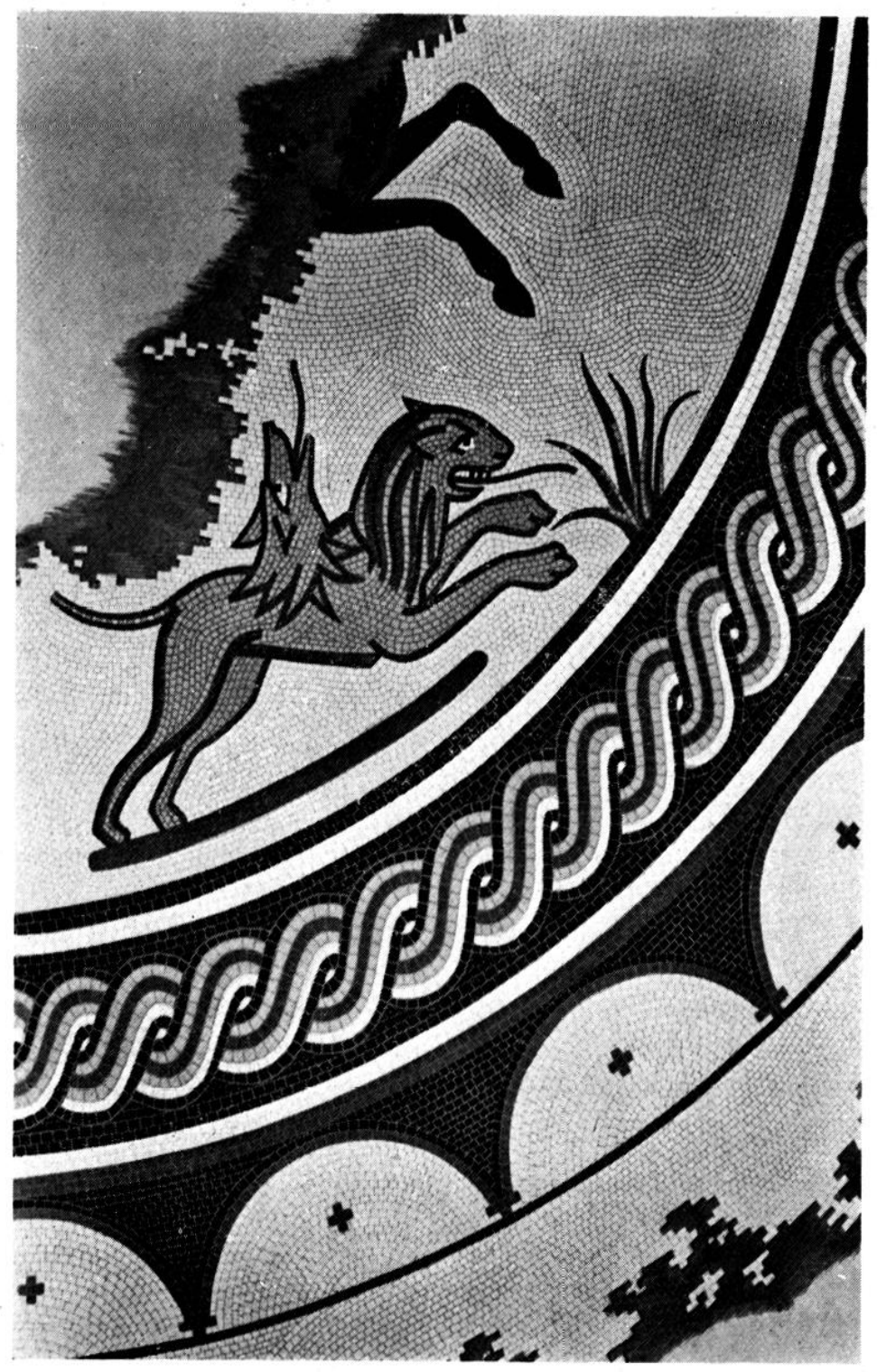

Firi. 1. - Coïmbre (Conimbriga), Portugal. Mosaïque de Bellérophon. Dessin.

rectement. Il a été mis au jour dans l'été de 1949, dans le triclinium d'une villa britto-romaine à Lullingstone (Kent), dans le Sud-Est de l'Angleterre; et il date de la première moitié du IV siècle $^{3}$.

(3) Publié par G.D.P. NicholsoN dans Archaeologia Cantiana, LXIII-1951, p. 44-47, pl. $1 b$ et $5 a, b$. Les photographies des mosaïques de Lullingstone sont reproduites ici grâce à l'aimable autorisation du Lieutenantcolonel G.IW. MEaTES, directeur des fouilles.
Ce triclinium est romposé de deux parties distinctes, mais étroitement liécs : à l'Est, un emplacement rarré dans le centre duquel s'étend la mosaïque au Bellérophon, composée de jolies tesserac, entourée de motifs géométriques d'une exécution beaucoup plus grossière; à l'Ouest, une abside semi-circulaire, dont le sol est un peu plus élevé que celui de la partie orientale, et que décore égale- 


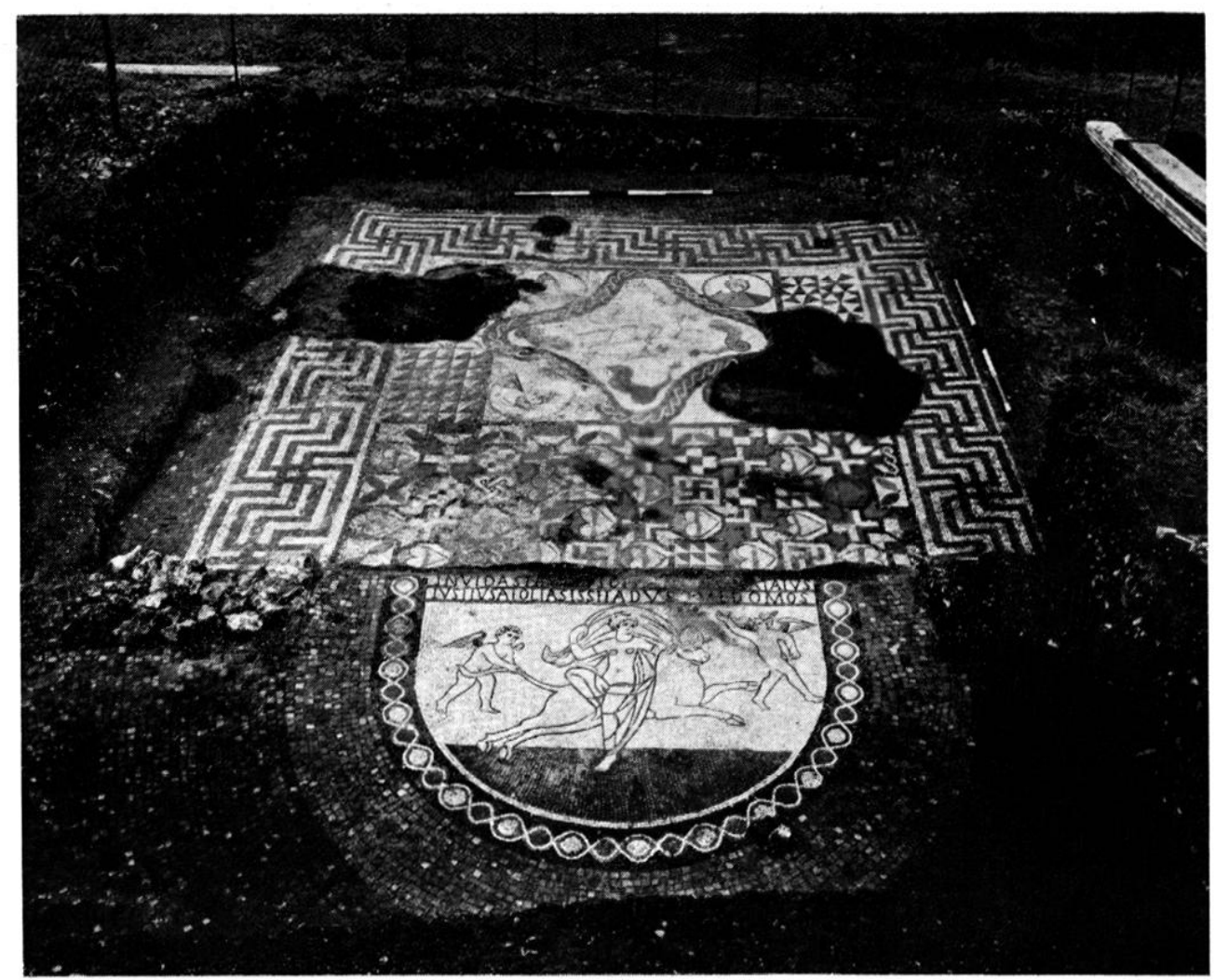

Fra. 2. - Lullingstone (Kent). Vue générale des deux mosaïques du triclinium.

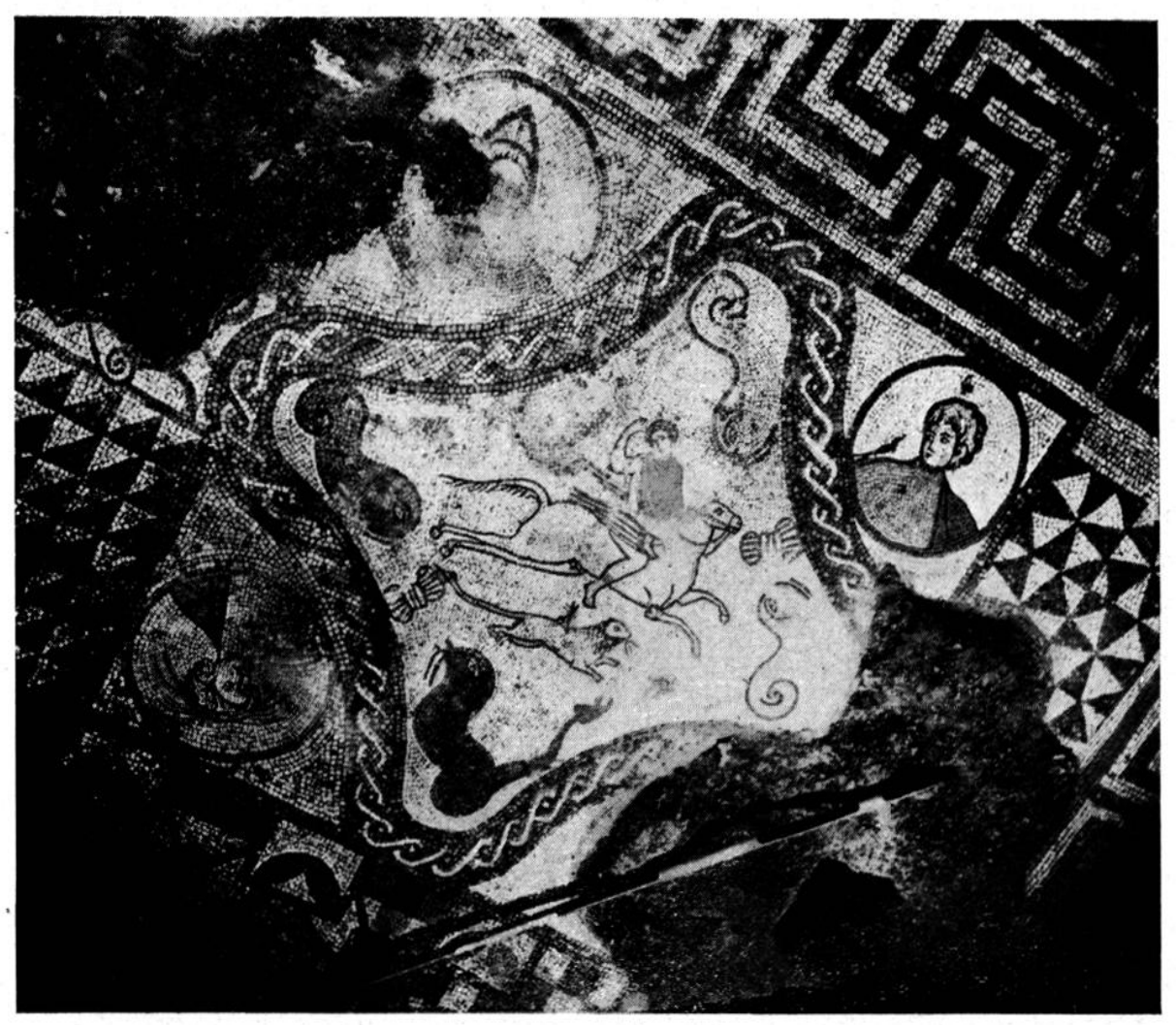

FIG. 3. - Lullingstone (Kent). La mosaïque de Belléroplion. 
ment une mosaïque figurée (fig. 2), représentant l'Enlèvement d'Europe, surmontée d'une inscription de deux lignes formant un distique élégiaque :

Invida si tiauri] uidisset Iuno natatus iustius Eolias isset adusque domos «Si Junon la Jalouse avait vu la nage du taureau, elle aurait eu plus d'excuses encore pour aller trouver tole en son palais », souvenir évident du livre premier de l'Énéide (v. 50-80), oì l'on voit Junon presser Éole de soulever une tempcte pour rattraper śnce. Autour du champ court une large bande de grosses tessères unies, sur laquelle on disposait la table en sigma et les lits des dîneurs. C'est pourquoi les deux mosaïques de la salle regardent vers le fond de l'abside 4 .

La disposition générale du Bellérophon de Lullingstone (fig. 3) est très semblable à celle de sa contrepartie nìmoise. Il est vrai qu'à Lullingstone le champ décoró n'est pas circulaire, mais en forme de «coussin», aux côtés incurvés, concaves. Mais dans l'un et l'autre pavements le cadre qui contient le panneau central est (arré, comme à Olynthe; et dans les coins du carré, à Iullingstone comme à Nîmes, il y a des bustes des Quatre Saisons, ceux de Lullingstone étant chacun dans un médaillon. L'Hiver porte un épais manteau à capuchon, costume adapté aux rigueurs du climat britannique et que l'on retrouve dans d'autres mosaïques représentant cette Saison, à Bignor (Sussex) et Chedworth (Gloucestershire); une hirondelle est perchée sur l'épaule du Printemps; l'Automne, qui paraît avoir usurpé la place occupée normalement par l'Été, a disparu; et l'Été, curieusement placé à còté de l'Hiver, est couronné d'épis.

(4) Cf. la mosaïque du triclinium dans la «Maison au Buffet» d'Antioche-sur-l'Oronte (Doro Levi, Antioch Mosaic Pavements, II, 191\%. pl. $33 a$ ).
Le groupe de Bellérophon (lig. 4), comme celui d'Europe, est dessiné au trait sur fond clair, sans ombres à l'infóricur. Les contours du premier sont noirs, ceux du second, rouges. Le héros porte un manteau et des chaussures rouges, tandis que le bois de la lance dont il perce la tête de chèvre de la Chimère est en cubes alternativement rouges et blancs. Cette tête de chìve est encore moins convaincante que celle de Nîmes; mais le souffle enflammé et la crinière du lion, ainsi que sa queue en forme de serpent, sont parfaitement reconnaissables. Dans chaque coin du « coussin 》 est un animal marin, probablement un dauphin; et à droite et à gauche de Pégase sont deux objets rayés, curieusement decrits dans Archaeologia Cantiana comme étant des «piliers »). Fn fait ce sont des coquillages bivalves, du genre «coquille Saint-Jacques», les deux valves, symétriques, étant ouvertes et relices par une charnière anormalement épaisse et massive. La comparaison avec les coquillages bivalves des pavements de Jurançon (Basses-Pyrénées, fig. 5) et de Dougga ('Tunisie, fig. 6) ne laisse aucun doute sur la correction de cette interprétation 6. Nous avons donc ici une association ctroite de Bellérophon avec l'eau, qui peut s'expliquer de deux façons. D'une part, la scène de Lullingstone peut illustrer la méthode de narration «simultanée », qui combine en un seul tableau deux épisodes successifs d'une mòme histoire : le héros traverse la mer en venant de Grèce et abat la Chimère en Lycie. J'autre part, les motifs aquatiques peuvent faire allusion au rôle de Bellérophon comme héros marin, par lequel M. Aymard explique la présence des oi-

(5) LXXIII-1951, p. 46.

(6) Inventaire des Mosaiques de la Gaule "f de l'Afrique: I, 1, Narbonnaise et Aquitaine, 109 : II. 1. Tunisie, 560. 


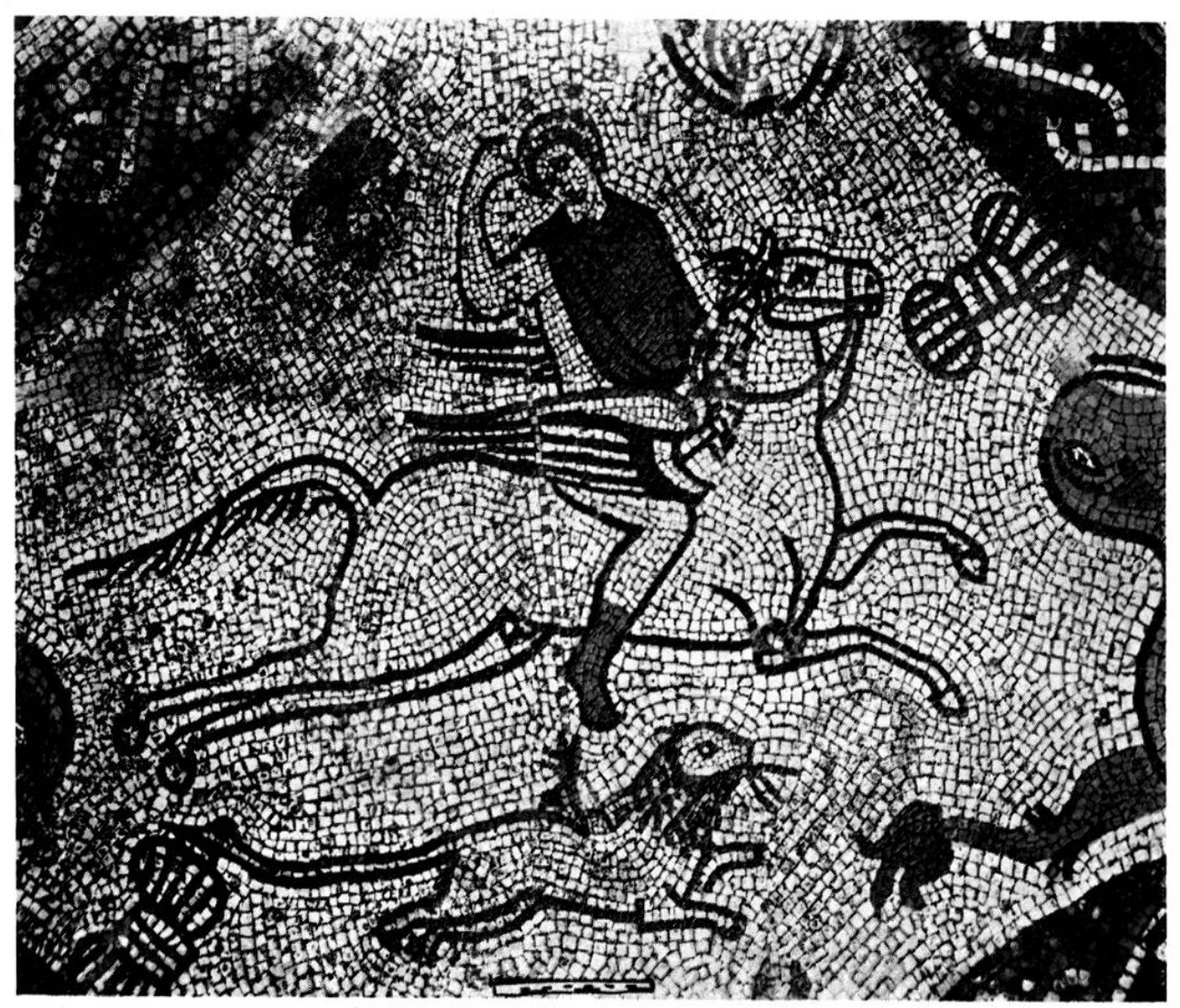

Fir. 4. - Lullingstone (Kent). Bellérophon. détail.

seaux aquatiques et des dauphins dans les panneaux secondaires de la mosaïque de Nîmes 7 .

Nous connaissons maintenant trois mosaïgues, Nîmes, Ravenne et lullingstone, oì la mise à mort de la Chimère par Bellérophon est associée avec les Saisons. Cette association ne peut être fortuite, ni purement «décorative». Elle doit avoir un sens : et reci pose la question de savoir quelles idées dictaient, sous l'Empire, le choix des sujets destinés aux pavements domestiques, problème auquel M. Aymard a apporté une si remarquable contribution dans sa publication de la mosaïque de Nîmes. Comme il le démontre, des thèmes tels que ceux de

(7) Op. cit., p. $26 \pi$.
Bellérophon et des Saisons peuvent s'inlerpréter dans le sens prophylactique, ou mor'al, ou eschatologique, ou les trois à la fois. Le Mal est banni de la demeure à laquelle le bien-ètre (les fruits des Saisons) est assuré; ou bien : les passions mauvaises sont surmontées et le bonheur confuis pour la maisonnée; ou bien : la mort est domptée, et une vie prospère et éternelle au paradis est acquise par le triomphe sur les puissances des ténèbres. A Lullingstone, où les mythes de Bellérophon et d'Europe sont réunis dans le même triclinium, le choix des sujets a dû être dicté principalement par des considérations eschatologiques. L'Enlèvement d'Europe n'est en effet ni prophylactique, ni moral... mais il évoque, comme celui de Ganymède, de Persépho- 


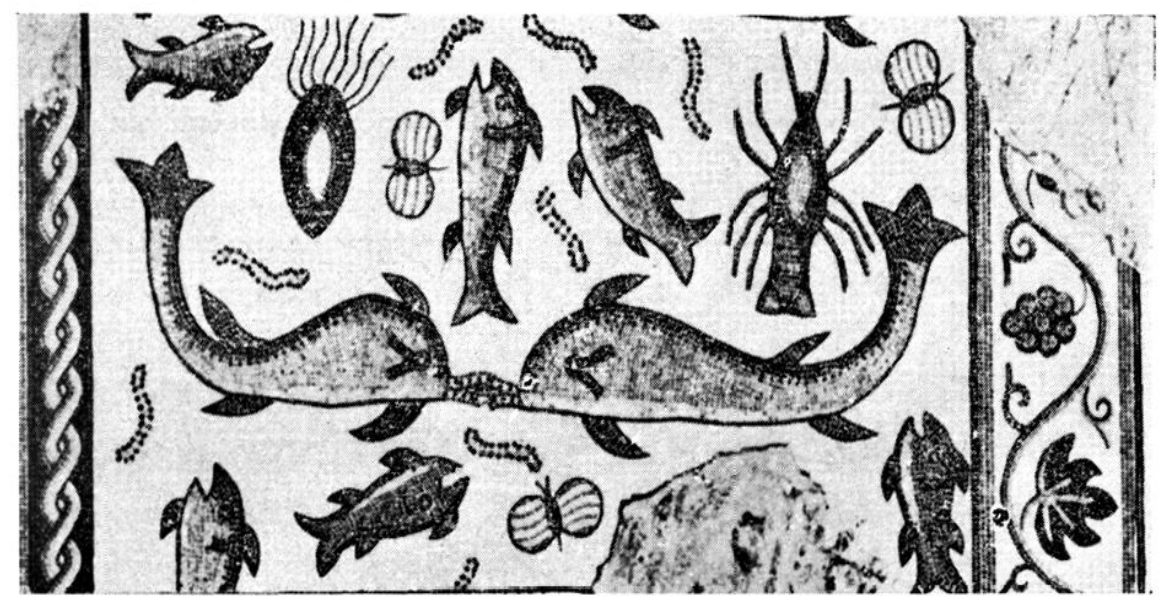

Fiti. 5. - Jurançon (Basses-Pyrénées). Mosiäque aquatique. Parlie de l'aqquarelle de l'Incentuire I, 1, Narbonnuise el .Aquilaine, n" 409.

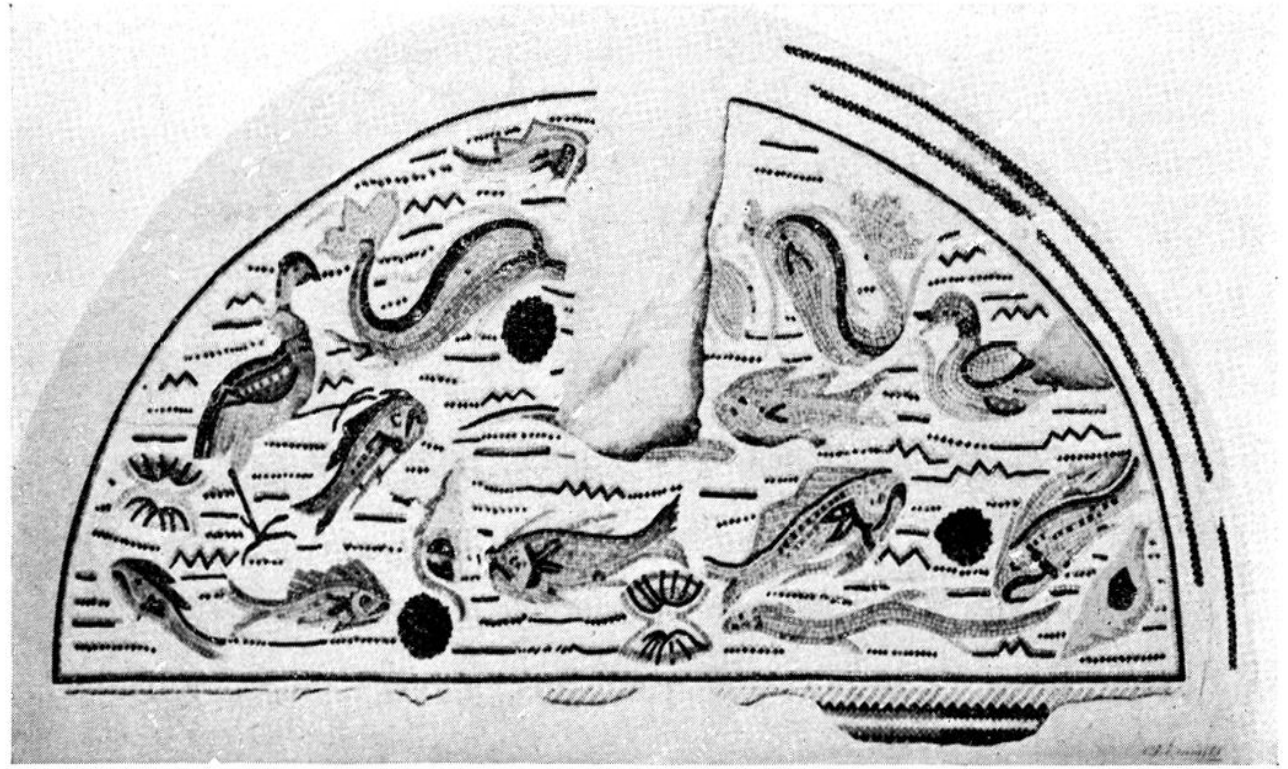

Fig. 6. - Dougga (Tunisie). Mosä̈que aquatique. Aquarelle de l'Incentaire, II, 1, Tunisie, $\mathrm{n}^{\circ} 56$ (6).

ne ou des Leucippides, l'âme « ravie » au corps au moment de la mort, et, pareil à celui du thiase marin, son voyage à traver's l'Océan ver's les Iles Fortunées. Ia nuance à demi-humoristique de la scène et du distique d'Furope, à Lullingstone, n'exclut pas ce genre de symbolisme sérieux, pas plus que ne le font les mines folâtres des joyeux monstres marins qui, sur les couvercles des sarcophages, emportent les âmes des défunts, par-dessus les vagues, jusqu'au paradis.

Dans l'esprit du paganisme grécoromain, la gravité et la gaîté se mêlaient aisément; et, à l'époque impériale, les croyances et les spéculations concernant 
la vie future n’étaient pas exprimées moins ouvertement, ni moins intimement mêlées à la trame de la routine quotidienne, que ne le sont la foi ot l'espoir des familles chrétiennes.

Jocelyn M.C. Torise.

Museum of Classical Archacolngy, Cambridge.

\section{Traduction P.-M. Duval.}

[Nous remercions vivement Miss ToYxBe, Professeur d'archéologie classique à l'Universile de Cambridge, de sa précieuse contribution aux problèmes soulevés par M. AYMand ¿a propos de la belle découverte de Nîmes. on ne manquera pas d'ètre frappé par le caractire si remarquable, a la fois original et somptueux, par le singulier intérèt et la date lardive des mosaïques britto-romaines. Et nous avons le plaisir d'annoncer qu'à l'occasion de l'enquête ouverte par l'Académie des Inscriptions en vue d'un Supplément à l'Inventaire des mosaïques de la Gaule, M. Henri STERn nous signale à Reims un nouveau pave-

ment au Bellérophon, encore inédit, qui porte à huit le nombre de ces représentations du héros. Sept proviennent donc des régions occidentales, où se confirme ainsi le succès durable, ou, pour reprendre les mots de II. AYMARD, «la renaissance discrète mais lenace, aux $\mathrm{II}^{\mathrm{e}}$ et $\mathrm{III}^{\circ}$ siècles, de la légende du héros corinthien et lycien».

$$
\text { P.-M. Duval. }
$$

\section{Plaqles-molgites Mérovingiennes DE LA FIN JU VII SIÈCLE}

Ie Musée de Isyde possède une plaqueboucle de bronze (Iongueur: $8^{\mathrm{rm}}$ ), trouvée à Garderen (Gueldre), dont W. Pleyte. a déjà publié un dessin dans son grand ouvrage illustré sur les antiquités des Paỵs-Bas 1, mais qui n'a pas encore reçu l'attention qu'clle mérite (fig. 1a). Flle étail assujetlie sur le cuir, non pas par des bossettes mobiles, mais par des lan-

(1) W. Pleyte, Nederlandsche Oudheden, Gelderland, pl. xx, 5 .

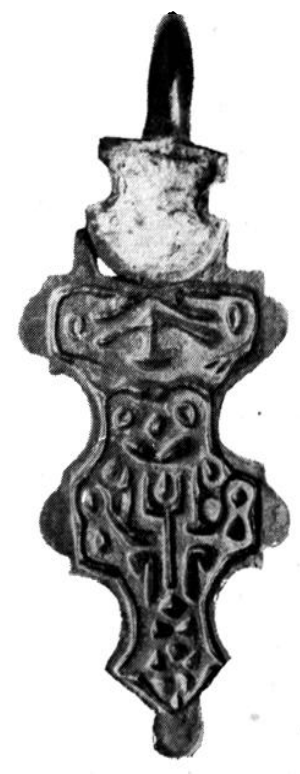

C

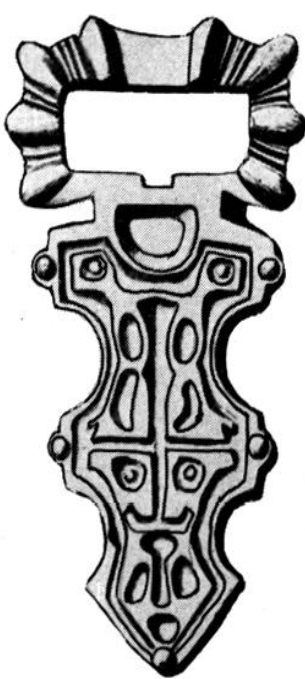

d

Fir. 1. - Plaques-boucles de Garderen (a), de l'Aisne $(b)$, du Iusée de Rouen (c), de Monceaux (Oise). Haut. de la plaque $a$, sans la boucle: $0^{\mathrm{m}}(1) 8$. (Réduction : un peu moins de la moitis;. 